\title{
Dynamics of thermoelastic frictional wear of a coating with piezoelectric interlayer
}

\author{
Polina A. Lapina ${ }^{1}$, Boris I. Mitrin ${ }^{1,}$, , Tatyana A. Kuznetsova ${ }^{2}$, and Vasilina A. Lapitskaya ${ }^{2}$ \\ ${ }^{1}$ Research and Education Center "Materials", Don State Technical University, 344000 Rostov-on- \\ Don, Russia \\ ${ }^{2}$ A.V. Luikov Heat and Mass Transfer Institute of the National Academy of Sciences of Belarus, \\ 220072 Minsk, Belarus
}

\begin{abstract}
To study dynamics of a coating wear, this paper deals with an uncoupled thermoelastic contact problem on sliding of a rigid punch over the surface of a thermoelastic coating bonded to an electroelastic interlayer, which itself is bonded to an undeformable substrate. Friction, frictional heating and coating wear on the contact is taken into account. The problem solution was constructed by the Laplace integral transform method and was obtained in the form of the Laplace convolutions. Based on the obtained expressions it is possible to develop technique for monitoring of a coating wear on a sliding contact through values of electric current arising in the piezoelectric interlayer.
\end{abstract}

\section{Introduction}

Coating of frictional pairs working surfaces are widely used to achieve different goals: reduce friction coefficient, corrosion resistance, thermal insulation and others. Frictional heating facilitate decrease of a coating lifetime and their breakdown. Coating wear modeling was previously performed in the framework of quasi-static thermoelasticity $[1,2]$. But interaction of thermoelastic extension and elastic dynamics during wear is usually not taken into account. Though, it was shown in $[3,4]$ and others that elastic dynamics has strong effect on thermoelastic contact parameters and stability conditions.

In [5], a methodology was proposed on indirect monitoring of sliding contact parameters by measuring electric current arising in a buried piezoceramic layer. The methodology is based on solution of a transient thermoelastodynamic contact problem. The purpose of the present paper is to obtain the solution of the similar contact problem incorporating wear of a coating.

\section{Problem statement}

Consider a transient dynamic contact problem of thermoelasticity and electroelasticity about sliding with a constant velocity of a rigid half-plane I over the surface of an elastic coating $A$ coupled along the lower face with an electroelastic non-thermal conducting

\footnotetext{
* Corresponding author: bmitrin@dstu.edu.ru
} 
layer $B$ whose polarization vector is orthogonal to it faces. On the lower boundary, the interlayer is adhered to a non-deformable substrate in the form of a half-plane II (see [5]). The elastic coating $A$ is exposed to abrasive wear by half-plane I indenting into its surface, while sliding parallel to the surface of the coating. The Coulomb friction and frictional heat generation at the coating interface is taken into account. The heat flux due to friction at the contact is directed into the elastic coating $A$. The edges of the electroelastic interlayer $B$ are covered with electrodes with an applied potential difference. At the initial moment, the displacements and their velocities in the coating and in the electroelastic interlayer are equal to zero, just like the initial temperature in the coating.

According to the formulation of the problem of deformation of a two-layer coating, all the basic physical parameters (temperature, stress, displacement, electric induction, electric field strength) do not depend on the choice of the horizontal coordinate and are functions only of the vertical coordinate and time. In this case, the behavior of the thermoelastic coating $A$ is described by a system of differential equations of thermoelasticity in conjunction with the heat equation [6]

$$
\begin{array}{cc}
\frac{\partial^{2} u}{\partial z^{2}}=\frac{3 \lambda+2 \mu}{\lambda+2 \mu} \alpha \frac{\partial T}{\partial z}, \quad a^{2}=\frac{2 \mu(1-v)}{\rho(1-2 v)} & 0<z<h, t>0 \\
\frac{\partial^{2} w}{\partial z^{2}}-\frac{1}{b^{2}} \frac{\partial^{2} w}{\partial t^{2}}=0, \quad b^{2}=\frac{\mu}{\rho} & 0<z<h, t>0 \\
\frac{\partial^{2} T}{\partial z^{2}}-\frac{1}{\kappa} \frac{\partial T}{\partial t}=0 & 0<z<h, t>0
\end{array}
$$

where $u(z, t), w(z, t)$ are vertical and horizontal displacements, $T(z, t)$ is the temperature distribution in the coating, $\lambda, \mu$ are Lamé parameters, $\rho, \kappa, \alpha, a, b$ are density, thermal diffusivity, linear thermal expansion coefficient, longitudinal and transverse elastic waves speed, of the coating material, correspondingly.

Duhamel - Neumann formulas [6] relate stresses, displacements and temperature between each other

$$
\sigma_{z z}=(\lambda+2 \mu) \frac{\partial u}{\partial z}-(3 \lambda+2 \mu) \alpha T, \quad \sigma_{x z}=\mu \frac{\partial w}{\partial z}
$$

where $\sigma_{z z}(z, t), \sigma_{x z}(z, t)$ are normal and tangential stresses in the coating.

Behavior of the electroelastic interlayer $B$ is described by a system of differential equations of electroelasticity for a piezoelectric material polarized in direction of $z$-axis [7]

$$
\begin{array}{ccc}
\frac{\partial^{2} u_{1}}{\partial z^{2}}-\frac{1}{a_{1}^{2}} \frac{\partial^{2} u_{1}}{\partial t^{2}}=0, a_{1}^{2}=\frac{c_{33}^{E}+c_{1}^{2} e_{33}}{\rho_{1}} & -H<z<0, t>0 \\
\frac{\partial^{2} w_{1}}{\partial z^{2}}-\frac{1}{b_{1}^{2}} \frac{\partial^{2} w_{1}}{\partial t^{2}}=0, & b_{1}^{2}=\frac{c_{44}^{E}}{\rho_{1}} & -H<z<0, t>0 \\
\frac{\partial^{2} \psi}{\partial z^{2}}=c_{1}{ }^{2} \frac{\partial^{2} u_{1}}{\partial z^{2}}, & c_{1}^{2}=\frac{e_{33}}{\varepsilon_{33}^{S}} & -H<z<0, t>0
\end{array}
$$

where $u_{1}(z, t), w_{1}(z, t)$ are elastic displacements in piezoelectric interlayer, $\psi(z, t)$ is electrostatic potential, $\rho_{1}$ is density of the interlayer material, $c_{33}^{E}, c_{44}^{E}$ are elastic moduli 
measured at constant current, $e_{33}$ is piezoelectric modulus, $\varepsilon_{33}^{S}$ is electric permittivity measured at constant deformation, $a_{1}, b_{1}$ are velocities of longitudinal and transverse elastic waves in the piezoelectric interlayer $B$. Stresses in the piezoelectric interlayer [7] are described by equations

$$
\sigma_{z z}^{1}=c_{33}^{E} \frac{\partial u_{1}}{\partial z}+e_{33} \frac{\partial \psi}{\partial z}, \quad \sigma_{x z}^{1}=c_{44}^{E} \frac{\partial w_{1}}{\partial z}
$$

where $\sigma_{\mathrm{zz}}^{1}(z, t), \sigma_{x z}^{1}(z, t)$ are normal and tangential stresses in the interlayer, are elastic moduli measured at constant current. Electric field intensity $E_{z}$, electric induction $D_{z}$ and electric current $I$ through the piezoelectric interlayer are determined from

$$
\begin{gathered}
E_{z}=-\frac{\partial \psi}{\partial z}, \quad D_{z}=\varepsilon_{33}^{S} E_{z}+e_{33} \frac{\partial u_{1}}{\partial z} \quad-H<z<0, t>0 \\
I=-S \frac{\partial D_{z}}{\partial t}
\end{gathered}
$$

where $S$ is cross-sectional area.

Mechanical, thermal and electrical boundary conditions of the formulated dynamic sliding contact problem are written as follows:

$$
\begin{array}{lll}
z=h & u(h, t)=-\Delta(t)+u_{\mathrm{w}}(t), & \sigma_{x z}(h, t)=-f \sigma_{z z}(h, t) \\
& & K \partial T(h, t) / \partial z=-f V \sigma_{z z}(h, t) \\
z=0 & u(0, t)=u_{1}(0, t), & w(0, t)=w_{1}(0, t) \\
& \sigma_{z z}(0, t)=\sigma_{z z}^{1}(0, t), & \sigma_{x z}(0, t)=\sigma_{x z}^{1}(0, t) \\
& T(0, t)=0, & \psi(0, t)=V_{0}(t) \\
z=-H & u_{1}(-H, t)=0 & w_{1}(-H, t)=0 \\
& \psi(-H, t)=-V_{0}(t) &
\end{array}
$$

where $u_{\mathrm{w}}(t)$ is the half-plane $I$ displacement due to coating wear, $f$ is coefficient of friction, $V$ is sliding velocity, $K$ is thermal conductivity of the coating $A, \Delta(t)$ is law of the half-plane $I$ penetration into the elastic coating, $h$ is the coating $A$ thickness, $H$ is the thickness of the piezoceramic interlayer $B, 2 V_{0}(t)$ is the electric potential difference between the interlayer electrodes. Here the abrasive wear model is used:

$$
u_{\mathrm{w}}(t)=-f V K^{*} \int_{0}^{t} \sigma_{x x}(h, \tau) d \tau, \quad t>0
$$

The problem statement is completed by zero initial conditions on displacements $u, w, u_{1}, w_{1}$, their velocities, temperature $T$, potential $\psi$ and penetration law $\Delta$ :

$$
\begin{gathered}
u(z, 0)=u_{1}(z, 0)=w(z, 0)=w_{1}(z, 0)=0 \\
\frac{\partial u(z, 0)}{\partial t}=\frac{\partial u_{1}(z, 0)}{\partial t}=\frac{\partial w(z, 0)}{\partial t}=\frac{\partial w_{1}(z, 0)}{\partial t}=0 \\
T(z, 0)=\psi(z, 0)=\Delta(0)=0
\end{gathered}
$$


In case of the formulated problem, vertical displacements in the coating $u(z, t)$ are independent from horizontal displacements $w(z, t)$. Horizontal displacements $w(z, t)$ can be determined from vertical displacements $u(z, t)$ after calculation of the latter. It is the reason why $w(z, t)$ are not considered in this paper.

\section{Exact solution of the problem}

By using the Laplace integral transform the solution of the formulated dynamic contact initial boundary value problem on sliding contact (1)-(19) can be written in form of the Laplace convolutions, which are Volterra integral equations of the considered problem:

$$
\begin{aligned}
& T(z, t)=\frac{1-v}{1+v} \frac{\hat{V}}{\alpha h}\left[\int_{0}^{t} \Delta(\tau) f_{T}^{0}(z, t-\tau) d \tau-\theta \int_{0}^{t} V_{0}(\tau) g_{T}^{0}(z, t-\tau) d \tau\right] \\
& u(z, t)=-\int_{0}^{t} \Delta(\tau) f_{u}^{0}(z, t-\tau) d \tau+\theta \int_{0}^{t} V_{0}(\tau) g_{u}^{0}(z, t-\tau) d \tau \quad 0 \leq z \leq h \\
& \sigma_{z z}(z, t)=-\frac{2 \mu(1-v)}{(1-2 \mu) h}\left[\int_{0}^{t} \Delta(\tau) f_{\sigma}^{0}(z, t-\tau) d \tau-\theta \int_{0}^{t} V_{0}(\tau) g_{\sigma}^{0}(z, t-\tau) d \tau\right] \quad 0 \leq z \leq h \\
& u_{1}(z, t)=-\int_{0}^{t} \Delta(\tau) f_{u_{1}}^{0}(z, t-\tau) d \tau+\theta \int_{0}^{t} V_{0}(\tau) g_{u_{1}}^{0}(z, t-\tau) d \tau \quad-H \leq z \leq 0 \\
& \sigma_{1 z z}(z, t)=-\frac{2 \mu(1-v)}{(1-2 \mu) h}\left[\int_{0}^{t} \Delta(\tau) f_{\sigma_{1}}^{0}(z, t-\tau) d \tau-\theta \int_{0}^{t} V_{0}(\tau) g_{\sigma_{1}}^{0}(z, t-\tau) d \tau+\right. \\
& \left.+\frac{2 \delta_{*}}{c_{1}^{2} H_{*}} V_{0}(t)\right] \\
& \psi(z, t)=-c_{1}^{2}\left(\int_{0}^{t} \Delta(\tau) f_{\psi}^{0}(z, t-\tau) d \tau-\theta \int_{0}^{t} V_{0}(\tau) g_{\psi}^{0}(z, t-\tau) d \tau\right)+ \\
& +\frac{2 z+H}{H} V_{0}(t) \\
& u_{w}(t)=\Delta(t)-\int_{0}^{t} \Delta(\tau) f_{u}^{0}(h, t-\tau) d \tau+\theta \int_{0}^{t} V_{0}(\tau) g_{u}^{0}(h, t-\tau) d \tau \\
& I_{0}(t)=-\frac{e_{33}}{H t_{\kappa}}\left[\int_{0}^{t} \Delta(\tau) f_{I}^{0}(z, t-\tau) d \tau-\theta \int_{0}^{t} V_{0}(\tau) g_{I}^{0}(z, t-\tau) d \tau\right]+\frac{2 \varepsilon_{33}^{S}}{H} \dot{V}_{0}(t) \\
& 0 \leq z \leq h
\end{aligned}
$$

where $I_{0}(t)=I(t) / S, f_{a}^{0}(z, t)$ and $g_{a}^{0}(z, t)$ are contour integrals:

$$
f_{a}^{0}(z, t)=\frac{1}{2 \pi \mathrm{i}} \int_{\Gamma} \frac{N_{a}(z, \zeta)}{t_{\kappa} R(\zeta)} e^{\zeta \tilde{t}} d \zeta, \quad g_{a}^{0}(z, t)=\frac{1}{2 \pi \mathrm{i}} \int_{\Gamma} \frac{M_{a}(z, \zeta)}{t_{\kappa} R(\zeta)} e^{\zeta \tilde{t}} d \zeta \quad \tilde{t}=\frac{t}{t_{\kappa}}
$$


Index $a$ is to be replaced with one of the following symbols: $T, u, \sigma, u_{1}, \sigma_{1}, \psi, I$. Components of integrands in (20)-(28) have the following form:

$$
\begin{aligned}
& N_{T}(z, \zeta)=\left(1-\gamma^{2} \zeta\right) \sqrt{\zeta} \sinh \left(\sqrt{\zeta} z h^{-1}\right) R_{1}^{*}(\zeta) \\
& M_{T}(z, \zeta)=\left(1-\gamma^{2} \zeta\right) \sqrt{\zeta} \sinh \left(\gamma \zeta a_{*} H_{*}\right) \sinh \left(\sqrt{\zeta} z h^{-1}\right) \\
& N_{u}(z, \zeta)=\gamma^{-1} m_{0}(\zeta) R_{3}(z, \zeta) \sinh \left(\gamma \zeta z h^{-1}\right)+R_{4}(z, \zeta) \cosh \left(\gamma \zeta z h^{-1}\right)-\hat{V} R_{1}^{*}(z, \zeta) \cosh \left(\sqrt{\zeta} z h^{-1}\right) \\
& M_{u}(z, \zeta)=R_{1}(\zeta) \gamma^{-1} \sinh \left(\gamma \zeta a_{*} H_{*}\right) \sinh \left(\gamma \zeta z h^{-1}\right)-\gamma^{-1} R_{4}^{*}(\zeta) \cosh \left(\gamma \zeta z h^{-1}\right) \\
& -\hat{V} \sinh \left(\gamma \zeta a_{*} H_{*}\right) \cosh \left(\sqrt{\zeta} z h^{-1}\right) \\
& N_{\sigma}(z, \zeta)=m_{0}(\zeta) R_{3}(\zeta) \zeta \cosh \left(\gamma \zeta z h^{-1}\right)+R_{4}(\zeta) \gamma \zeta \sinh \left(\gamma \zeta z h^{-1}\right)-\hat{V} R_{1}^{*}(\zeta) \gamma^{2} \zeta \sqrt{\zeta} \sinh \left(\sqrt{\zeta} z h^{-1}\right) \\
& M_{\sigma}(z, \zeta)=R_{1}(\zeta) \zeta \sinh \left(\gamma \zeta a_{*} H_{*}\right) \cosh \left(\gamma \zeta z h^{-1}\right)-R_{4}^{*}(\zeta) \zeta \sinh \left(\gamma \zeta z h^{-1}\right) \\
& -\hat{V} \gamma^{2} \zeta \sqrt{\zeta} \sinh \left(\gamma \zeta a_{*} H_{*}\right) \sinh \left(\sqrt{\zeta} z h^{-1}\right) \\
& (0 \leq z \leq h) \\
& N_{u_{1}}(z, \zeta)=H_{*} \zeta R_{3}(\zeta) \sinh \left(\gamma \zeta a_{*} H_{*}\left(z h^{-1} H_{*}^{-1}+1\right)\right) \\
& M_{u_{1}}(z, \zeta)=-\gamma^{-1} R_{0}(\zeta) \sinh \left(\gamma \zeta a_{*} H_{*}\left(z h^{-1} H_{*}^{-1}+1\right)\right) \\
& N_{\sigma_{1}}(z, \zeta)=\zeta l_{1}(z, \zeta) R_{3}(\zeta) \quad M_{\sigma_{1}}(z, \zeta)=-\gamma^{-1} H_{*}^{-1} l_{1}(z, \zeta) R_{0}(\zeta) \\
& N_{\psi}(z, \zeta)=H_{*} \zeta l_{0}(z, \zeta) R_{3}(\zeta) \\
& M_{\psi}(z, \zeta)=-\gamma^{-1} l_{0}(z, \zeta) R_{0}(\zeta) \\
& l_{0}(z, \zeta)=\sinh \left(\gamma \zeta a_{*} H_{*}\left(z h^{-1} H_{*}^{-1}+1\right)\right)-\sinh \left(\gamma \zeta a_{*} H_{*}\right) \cdot\left(z h^{-1} H_{*}^{-1}+1\right) \\
& l_{1}(z, \zeta)=\delta a_{*} H_{*} \gamma \zeta \cosh \left(\gamma \zeta a_{*} H_{*}\left(z h^{-1} H_{*}^{-1}+1\right)\right)-\delta_{*} \sinh \left(\gamma \zeta a_{*} H_{*}\right) \\
& (-H \leq z \leq 0) \\
& N_{I}(\zeta)=-H_{*} \zeta^{2} R_{3}(\zeta) \sinh \gamma \zeta a_{*} H_{*} \\
& M_{I}(\zeta)=\gamma^{-1} \zeta R_{0}(\zeta) \sinh \gamma \zeta a_{*} H_{*} \\
& R(\zeta)=m_{0}(\zeta) \gamma^{-1} R_{0}(\zeta)+\zeta H_{*} R_{1}(\zeta) \sinh \left(\gamma \zeta a_{*} H_{*}\right) \\
& m_{0}(\zeta)=\delta \cosh \left(\gamma \zeta a_{*} H_{*}\right) \gamma \zeta a_{*} H_{*}-\delta_{*} \sinh \left(\gamma \zeta a_{*} H_{*}\right) \\
& R_{0}(\zeta)=\left(1-\gamma^{2} \zeta\right) \cosh \sqrt{\zeta} \sinh \gamma \zeta-\gamma \hat{V}\left(\left(1-\left(1-\gamma^{2} \zeta\right) k_{w}\right) \cosh \sqrt{\zeta} \cosh \gamma \zeta\right. \\
& -\gamma \sqrt{\zeta} \sinh \sqrt{\zeta} \sinh \gamma \zeta-1) \\
& R_{1}(\zeta)=\left(1-\gamma^{2} \zeta\right) \cosh \sqrt{\zeta} \cosh \gamma \zeta-\gamma \hat{V}\left(\left(1-\left(1-\gamma^{2} \zeta\right) k_{w}\right) \cosh \sqrt{\zeta} \sinh \gamma \zeta\right) \\
& -\gamma \sqrt{\zeta} \sinh \sqrt{\zeta} \cosh \gamma \zeta) \\
& R_{1}^{*}(\zeta)=H_{*} \gamma \zeta \sinh \left(\gamma \zeta a_{*} H_{*}\right) \sinh \gamma \zeta+m_{0}(\zeta) \cosh \gamma \zeta \\
& R_{3}(\zeta)=\left(1-\gamma^{2} \zeta\right) \cosh \sqrt{\zeta}-\gamma \hat{V}(\sinh \gamma \zeta-\gamma \sqrt{\zeta} \sinh \sqrt{\zeta}) \\
& R_{4}(\zeta)=\zeta H_{*}\left(1-\gamma^{2} \zeta\right) \sinh \left(\gamma \zeta a_{*} H_{*}\right) \cosh \sqrt{\zeta}+ \\
& \gamma \hat{V}\left(H_{*} \gamma \zeta \sqrt{\zeta} \sinh \sqrt{\zeta} \sinh \left(\gamma \zeta a_{*} H_{*}\right)+m_{0}(\zeta) \gamma^{-1} \cosh \gamma \zeta\right) \\
& R_{4}^{*}(\zeta)=\left(R_{0}(\zeta)-\gamma \hat{V}\right) \sinh \left(\gamma \zeta a_{*} H_{*}\right)
\end{aligned}
$$


where

$$
\begin{gathered}
t_{\kappa}=\frac{h^{2}}{\kappa}, \eta=\frac{H_{*}}{\delta_{0}}, \delta=\delta_{0}+\delta_{*}, \delta_{0}=\frac{c_{33}^{E}}{\lambda+2 \mu}, \delta_{*}=\frac{c_{1}^{2} e_{33}}{\lambda+2 \mu}, \\
\gamma=\frac{\kappa}{a h}, \hat{V}=\frac{f V \alpha}{K} \frac{2 \mu(1+v) h}{1-2 v}, a_{*}=\frac{a}{a_{1}}, H_{*}=\frac{H}{h}, \theta=\frac{2 \delta_{*}}{c_{1}^{2}}=\frac{2 e_{33} \delta_{0}}{c_{33}^{E}}
\end{gathered}
$$

$\Gamma=\left\{\zeta:-i \infty+d t_{\kappa}, i \infty+d t_{\kappa}\right\}$ is the contour of integration, where $d$ is defined in a way to keep all of isolated singularities of integrands of (28) (derived by (32)) to the left of the contour $\Gamma, v=\lambda /(2(\lambda+\mu))$ is the Poisson's ratio of the coating $A$.

Investigation of integrands of (28) shows that they are all meromorphic in the complex plane of the integration variable $\zeta=\xi+i \eta$ and have only poles as their isolated singularities. To effectively calculate the contour integrals, the investigation of poles of their integrands should be carried out, similar to $[2,4,5]$.

\section{Conclusion}

The obtained formulas allow to calculate wear of an elastic coating and also electic current arising in the piezoelectric interlayer during the engagement, along with other contact characteristics, using the approach of [3] for calculating the integrals (20)-(27). This extends the methodology of contact parameters monitoring proposed in [5] to the case when abrasive wear of the coating takes place.

P.A. Lapina and B.I. Mitrin was supported by the State Assignment of the Ministry of Education and Science of Russia no. 9.1481.2017/4.6 and Russian Foundation for Basic Research grants no. 18-0701397-a, 17-57-04065-Bel_mol_a. V.A. Lapitskaya and T.A.Kuznetsova was supported by the Belarusian Republican Foundation for Fundamental Research grants no. F17RM-021 and no. F18R-239.

\section{References}

1. A.A. Evtushenko, Yu.A. Pyryev, Izvestiya RAN. Mekhanika Tverdogo Tela (1), 114 (1997)

2. V.B. Zelentsov, B.I. Mitrin, I.A. Lubyagin, Comp. Cont. Mech. 9(4), 430 (2016)

3. L. Afferrante, M. Ciavarella, J.R. Barber, Proc. R. Soc. A 462, 2161 (2006)

4. V.B. Zelentsov, B.I. Mitrin, S.M. Aizikovich, J. Frict. Wear 37(3), 213 (2016)

5. V.B. Zelentsov, B.I. Mitrin, S.M. Aizikovich, The 2017 World Congress on Advances in Structural Engineering and Mechanics (Techno-Press, Ilsan, 2017)

6. A.D. Kovalenko, Introduction to Thermoelasticity (Naukova Dumka, Kiev, 1965)

7. V.T. Grinchenko, A.F. Ulitko, N.A. Shul'ga, Electroelasticity (Naukova Dumka, Kiev, 1980) 